\title{
HRTEM Image Simulations for the Study of Ultra-Thin Gate Oxides
}

(Brief Title: HRTEM Simulations)

\author{
Seth T. Taylor ${ }^{1,3}$, John Mardinly ${ }^{2}$, and Michael A. O'Keefe ${ }^{3}$ \\ ${ }^{1}$ Department of Materials Science and Engineering \\ University of California \\ Berkeley, CA 94720 \\ ${ }^{2}$ Intel Corporation \\ Materials Technology Department \\ Santa Clara, CA 95052 \\ ${ }^{3}$ National Center for Electron Microscopy \\ Lawrence Berkeley National Laboratory \\ Berkeley, CA 94720
}

Corresponding Author: Seth Taylor

577 Evans Hall

Materials Science and Engineering

University of California

Berkeley, CA 94720

TEL: (510) 486-4590

FAX: (510) 486-5888

Email: sttaylor@ socrates.berkeley.edu 


\section{$\underline{\text { Abstract }}$}

We have performed high resolution transmission electron microscope (HRTEM) image simulations to qualitatively assess the visibility of various structural defects in ultra-thin gate oxides of MOSFET devices, and to quantitatively examine the accuracy of HRTEM in performing gate oxide metrology. Structural models contained crystalline defects embedded in an amorphous $16 \AA$-thick gate oxide. Simulated images were calculated for structures viewed in cross-section. Defect visibility was assessed as a function of specimen thickness and defect morphology, composition, size and orientation. Defect morphologies included asperities lying on the substrate surface, as well as "bridging" defects connecting the substrate to the gate electrode. Measurements of gate oxide thickness extracted from simulated images were compared to actual dimensions in the model structure to assess TEM accuracy for metrology. The effects of specimen tilt, specimen thickness, objective lens defocus and coefficient of spherical aberration $\left(\mathrm{C}_{\mathrm{s}}\right)$ on measurement accuracy were explored for nominal $10 \AA$ gate oxide thickness. Results from this work suggest that accurate metrology of ultra-thin gate oxides (i.e. limited to several per cent error) is feasible on a consistent basis only by using a $\mathrm{C}_{\mathrm{s}}$-corrected microscope. However, fundamental limitations remain for characterizing defects in gate oxides using HRTEM, even with the new generation of $\mathrm{C}_{\mathrm{s}}$-corrected microscopes.

Key words: HRTEM image simulations, metrology, gate oxides, defect analysis 


\section{Introduction}

High-resolution transmission electron microscopy is used extensively in the semiconductor industry for device characterization, and has become one of the highly favored techniques for characterizing the latest generation of ultra-thin gate oxides in MOSFET devices. Despite growing use of HRTEM for the study of metrology and crystal defects in gate oxides, very little information has been gleaned regarding the limitations of HRTEM in assessing these parameters. As gate oxide thickness approaches $10 \AA$ or less, the ability of HRTEM to detect structural defects in gate oxides, to perform metrology, or to measure planarity to within a few per cent accuracy, remains surprisingly unclear. This uncertainty stems from an inability to perform experimental HRTEM studies of gate oxides in a controlled fashion. To investigate model defects experimentally, one would have to construct gate oxides with structural defects whose size and morphology are known perfectly a priori, successfully make thin specimens that capture the defects, and then perform imaging experiments in the HRTEM to assess defect visibility. Since this task is virtually impossible, alternate techniques utilizing HRTEM image simulations must be employed to assess the limitations of experimental HRTEM for gate oxide metrology and characterization.

In this study, we performed HRTEM image simulations of a MOSFET device to investigate the ability of the HRTEM to "detect" various structural defects in gate oxides, and to quantify the accuracy of HRTEM in measuring gate oxide thickness.

\section{Materials and Methods}

All image simulations were performed reproducing optimum imaging conditions for a JEOL 2010F HRTEM operating at $200 \mathrm{keV}$. Relevant instrumental parameters required as input 
for the image simulation software include the objective lens $\mathrm{C}_{\mathrm{s}}(=0.5 \mathrm{~mm})$, the convergence angle ( $\alpha=0.55 \mathrm{mrad})$ of the incident electrons, and the spread of defocus $(\Delta=30 \AA)$, a conglomerate term which accounts for fluctuations in electron energies and voltage instabilities. Model crystal structures were assembled using CrystalKit ${ }^{\mathrm{TM}}$ software, and subsequent image simulations performed using the MacTEMPAS ${ }^{\text {TM }}$ software derived from NCEMSS (O'Keefe and Kilaas, 1988). The simulation software utilizes the multislice approach pioneered by Cowley and Moodie (1957; Lynch, 1971; Goodman and Moodie, 1974) to model the three dimensional potential of the specimen and calculate an exit surface wavefunction for a planar incident electron wavefront .

The MOSFET device was modeled as a thin gate oxide separating a (100) silicon substrate from the gate electrode. Though typical gates of real devices are fabricated using polysilicon, we used single crystal (100) silicon for the gate electrode to facilitate construction of a model structure. The gate oxide, an amorphous silicon oxide with a mass density of approximately $2.4 \mathrm{~g} / \mathrm{cm}^{3}$, was generated using a FORTRAN routine that preserves Si-O bond angles and $\mathrm{SiO}_{2}$ stoichiometry. Interfaces between the gate oxide and the adjoining silicon were atomically sharp, with no introduced roughness and no gradient in oxygen concentration perpendicular to the $\mathrm{Si}_{-} \mathrm{SiO}_{2}$ interface. Image simulations of the device structure in cross-section were performed with Si[110] parallel to the electron beam direction (see Fig 1). The cell thickness for the multislice calculation was taken to be $7.68 \AA$, or one unit cell distance in the $\operatorname{Si}[110]$ direction. A layer of amorphous silicon was added to the top and bottom of the specimen to account for amorphization that is commonly known to occur during typical specimen preparation. 


\section{Defect Studies}

We modeled crystalline defects embedded in a $16 \AA$ amorphous gate oxide. Images were calculated with varying defect morphology, composition, size and orientation. We considered both hemispherical and pyramidal asperities lying on the substrate surface, as well as "bridging" defects that connect the substrate to the gate electrode. Bridging defects include crystalline silicon defects of various sizes having cylindrical morphologies, and oriented with different lowindex directions parallel to the beam direction. Surface defects include small silicon asperities oriented along low-index directions. The total specimen thickness in the beam direction varied from $92 \AA$ to upwards of $300 \AA$ in order to understand the effect of specimen thickness on defect visibility within the allowable thickness range for HRTEM image simulations. Defect visibility was assessed qualitatively according to detection of periodic contrast in the amorphous gate oxide, stemming from the crystallinity of the defect structure.

\section{Metrology Studies}

Measurements of gate oxide thickness extracted from simulated images of non-defect structures were compared to actual dimensions in the model structure to assess TEM accuracy. By using simulated images, we significantly reduce the error associated with determining the gate oxide boundaries (since these can be precisely located on the model), and can therefore measure the error introduced solely by the HRTEM imaging process. The actual gate oxide thickness, T, is defined as the distance between the bounding Si atoms in the model structure (Fig. 2a), while the observed gate oxide thickness (t) is measured directly in pixels from the simulated image (Fig. 2b). We used a statistical routine to calculate the standard deviation in pixel intensity for each horizontal row (or y-coordinate) in the simulated image (Fig. 2c). Local 
minima in the standard deviation, which correspond to low-intensity regions between $\mathrm{Si}[110]$ columns, and local maxima corresponding to the centers of columns, serve to calibrate the image length scale. The measured gate oxide thickness $(\mathrm{t})$ is then compared to the actual thickness $(\mathrm{T})$ to assess measurement accuracy for a variety of microscope and specimen conditions. The effects of specimen tilt, specimen thickness, objective lens defocus and coefficient of spherical aberration $\left(\mathrm{C}_{\mathrm{s}}\right)$ on measurement accuracy were explored for nominal $10 \AA$ gate oxides.

\section{$\underline{\text { Results }}$}

The reader is reminded that all images presented here are derived from multislice calculations of model structures, and are not experimental HRTEM images.

\section{Defect Studies}

Intuitively, one might expect defect visibility to scale directly with defect size, and to scale inversely with specimen thickness. These trends were confirmed by simulations performed for various defect structures embedded in a $16 \AA$ gate oxide. Fig. 3 shows the effect of defect size on visibility for a [110]-oriented silicon defect modeled as a cylinder bridging the substrate and gate electrode. At a specimen thickness of $138 \AA$, the $20 \AA$-diameter cylindrical defect is faintly detectable (Fig. 3a), whereas the $10 \AA$ defect cannot be discerned (Fig. 3b). Defect visibility is observed to scale directly with defect size in Figure 4, which shows simulated images for [110]-oriented Si hemispherical defects protruding from the substrate surface. Here, visibility decreases sharply as the hemisphere radius shrinks from $16 \AA$ (Fig. 4 a) to $13 \AA$ (Fig.

4b) to $10 \AA$ (Fig 4c) at a constant specimen thickness of $123 \AA$.

The effect of crystal orientation on defect visibility is illustrated in Fig. 5 for hemispherical surface defects with $16 \AA$ radius. At $123 \AA$ specimen thickness, the [110]-oriented 
Si defect is slightly visible (Fig. 4a), the [111] defect is less visible (Fig. 5a), and the [100] defect is effectively invisible (Fig. 5b). A similar trend is observed for cylindrical Si defects in which the [110]-oriented defect exhibits the greatest visibility, while [111]- and [100]-oriented defects display significantly reduced visibilities at the same specimen thickness. Simulations show that for a specimen thickness of $138 \AA$, the cylinder (defect) diameter must be in excess of $30 \AA$ for the [111]-oriented Si defect to be detected, and greater than $40 \AA$ for detection of the [100]oriented Si defect.

In an effort to improve the visibility of [111]- and [100]-oriented defects, we performed image simulations of defect structures using reduced values of objective lens $C_{s}$ to extend the microscope resolution. Table I lists the modified $\mathrm{C}_{\mathrm{s}}$ values, in addition to the relevant Scherzer defocus values and point-to-point resolutions (as measured from first crossover in CTF) calculated for the JEOL 2010F HRTEM used in this study. Optimum defocus, $\varepsilon$, for $\mathrm{C}_{\mathrm{s}}=0.0$ $\mathrm{mm}$ was determined according to the expression (O'Keefe, 1998)

$$
\chi(\mathbf{u})=\pi \varepsilon \lambda|\mathbf{u}|^{2}
$$

where $\chi(\mathbf{u})$ is the phase change, $\mathbf{u}$ is the spatial frequency, and $\lambda$ is the electron wavelength. Resolution is optimized by constraining the phase change to not exceed $\pi / 2$ (O'Keefe, 1998). The optimum defocus for $\mathrm{C}_{\mathrm{s}}=0.0 \mathrm{~mm}$ reported in Table I is for a spatial frequency of $1.0 \AA$ at CTF crossover.

Figures 6 and 7 show image simulations for [111]- and [100]-oriented Si hemispherical defects with $16 \AA$ radius, respectively, at a specimen thickness of $123 \AA$. For $\mathrm{C}_{\mathrm{s}}=0.2 \mathrm{~mm}$ (Figs. 6a and 7a) and $\mathrm{C}_{\mathrm{s}}=0.05 \mathrm{~mm}$ (Figs. $6 \mathrm{~b}$ and $7 \mathrm{~b}$ ), defect visibility is marginally improved relative to that observed for $\mathrm{C}_{\mathrm{s}}=0.5 \mathrm{~mm}$ (see Figs. $5 \mathrm{~b}$ and $5 \mathrm{c}$ ). Even at $\mathrm{C}_{\mathrm{s}}=0.0 \mathrm{~mm}$, the [100]-oriented defect (Fig. 7c) does not exhibit any marked increase in visibility. However, the [111]-oriented 
defect (Fig. 6c) is readily discerned in the gate oxide at zero $\mathrm{C}_{\mathrm{s}}$, with the familiar hexagonal arrangement of atoms apparent in the magnified view of Fig. 6d. Surprisingly, simulated images of [110]-oriented defects using these same reduced $C_{s}$ values show no improvements in defect visibility.

Large cylindrical voids, as well as amorphous Si defects up to $30 \AA$ in diameter, were also incorporated in the gate oxide, but these defects were not visible at practical specimen thicknesses examined here. Finally, it should be noted that images simulated at Scherzer defocus consistently yielded greater visibility of defect structures than those simulated at minimum contrast defocus.

\section{Metrology Studies}

Table II summarizes the measurement errors encountered for all imaging conditions examined in this study. In contrast to the defect studies, where Scherzer defocus proved to be a more advantageous imaging condition than minimum contrast, metrology results reveal no consistent improvements in measuring error for Scherzer defocus relative to minimum contrast. In fact, minimum contrast defocus is observed to yield greater accuracy than Scherzer defocus for at least one specimen thickness (compare Experiments 11 and 12 from Table II).

Images simulated with varying degrees of specimen tilt, introduced about a tilt axis perpendicular to the gate oxide thickness, show no consistent trends in measurement accuracy as a function of tilt angle. This is demonstrated for $10 \AA$ gate oxides imaged at Scherzer defocus in Fig. 8 for two different specimen thicknesses. Similarly, images simulated with different spherical aberration coefficients exhibit no obvious relationship between measurement accuracy and $\mathrm{C}_{\mathrm{s}}$, as shown in Figure 9 for $10 \AA$ gate oxides and $154 \AA$ specimen thickness. 
Completely correcting for spherical aberration in the objective lens results in consistent measurement accuracy of gate oxide thickness, regardless of specimen thickness and tilt. Figure 10 shows $\mathrm{C}_{\mathrm{s}}$-corrected images simulated at Scherzer defocus for two different specimen thicknesses, $154 \AA$ (Fig. 10a) and $246 \AA$ (Fig. 10b). The former has a measurement error of $-1.1 \%$, while the latter, despite an inability to resolve individual atoms in the Si dumbbell, exhibits no error at all. As we introduce varying amounts of specimen tilt, the $\mathrm{C}_{\mathrm{s}}$-corrected image still provides very accurate measurements of gate oxide thickness, as shown in Figure 11

for $154 \AA$ thick specimens. Here it is observed that specimen tilts as large as $12.6 \mathrm{mrad}$ result in errors of approximately $1 \%$ for $\mathrm{C}_{\mathrm{s}}=0.0 \mathrm{~mm}$.

\section{Discussion}

Defect visibility appears to be governed primarily by defect orientation and the projected thickness of the defect in the beam direction. Simulations show that $\mathrm{Si}[110]$ is the most visible defect orientation, regardless of defect size or morphology. This result is consistent with the spacing of Si atoms in the three different low-index projections. Silicon [111] and [100] defects could only be observed at very small specimen thicknesses $(<100 \AA)$ or for very large defect sizes

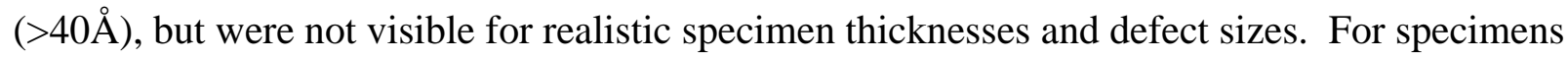
significantly thicker than $250 \AA$, which is typical of routine experimental work, crystalline defects less than $\approx 30 \AA$ in size become invisible regardless of orientation. If HRTEM is to be successfully utilized for detecting and characterizing structural defects in gate oxides, specimen preparation routines will have to be significantly improved to consistently yield thin crosssectional foils with $200 \AA$ A thickness. Recent studies (Jamison, 2000) show that the total amorphized damage introduced by Ga+ focussed ion beams is alone greater than $200 \AA$, 
suggesting that current FIB-based preparation routines, favored by many in the semiconductor industry, are not amenable to the study of such small defects.

Ongoing efforts to correct spherical aberration of the objective lens (Haider et al., 1998; Urban et al., 1999) have been closely watched by the semiconductor industry with hopes that a new generation of $\mathrm{C}_{\mathrm{s}}$-corrected microscopes might improve the current status of HRTEM-based device characterization. Results from our work suggest that improvements in gate oxide metrology can be expected with $\mathrm{C}_{\mathrm{s}}$-corrected microscopes. The ability to perform accurate metrology (to within 1\% error) in thick samples with sizeable specimen tilt is truly an intriguing result. However, gate oxide defect detection and characterization may not experience such fruitful gains. As we have demonstrated, reducing the microscope's coefficient of spherical aberration to zero enhances the visibility of [111]-oriented silicon defects, but does not noticeably improve the visibility of [100] or [110] silicon defects.

Results from the metrology studies reveal no consistent trends in measurement accuracy as a function of specimen thickness, specimen tilt, or objective lens defocus. Previous work has shown that while Scherzer defocus yields the most accurate representation of the projection of the specimen potential for a thin specimen, the best defocus for accurate projection of thicker structures moves steadily in the overfocus direction as specimen thickness is increased (O'Keefe et al., 1989). This may help to explain why measurement errors for Scherzer defocus are sometimes greater than those for minimum contrast defocus, particularly for thicker ( $246 \AA$ ) specimens.

The fact that specimen tilt can improve measurement accuracy, as observed in Fig. 8, can perhaps be understood in light of previous studies by O'Keefe and Radmilovic (1992; 1993). These authors demonstrate that "thin crystal" character in simulated images can be preserved at 
large specimen thicknesses by introducing small amounts of specimen tilt. As a result of tilting, the projected potential of each atomic column is reduced and effectively smeared out, causing the weak phase object approximation to be satisfied to much greater (overall) specimen thicknesses than with no tilt. The net effect is for the crystal to appear thinner in the simulations, which may explain why specimen tilt doesn't necessarily harm measurement accuracy of gate oxide thickness deduced from multislice calculations. Additionally, the process of introducing specimen tilt along an axis parallel to the gate oxide thickness diffuses (as seen by the electron beam) the abrupt, unnaturally sharp $\mathrm{Si}-\mathrm{SiO}_{2}$ interface. Consequently, the pronounced Fresnel diffraction that occurs at this interface is reduced, yielding more accurate measurements of gate oxide thickness. Future studies of gate oxide metrology need to incorporate some degree of interface roughness when modeling the $\mathrm{Si}-\mathrm{SiO}_{2}$ interface. Modified interface structures which more closely capture the real structure of the $\mathrm{Si}_{-} \mathrm{SiO}_{2}$ interface should help to quantify accuracy for gate oxides likely to be encountered in practice.

\section{Conclusions}

Results from this work reveal some fundamental limitations in HRTEM for characterizing defects in gate oxides, even with the new generation of $\mathrm{C}_{\mathrm{s}}$-corrected microscopes. While zero- $\mathrm{C}_{\mathrm{s}}$ imaging may prove to be advantageous in "resolving" certain structural defects in gate oxides, it does not appear to be universally propitious for all defects. Meanwhile, preparation of thin foil specimens continues to be a major obstacle to HRTEM-based identification of gate oxide defects. Studies of gate oxide thickness suggest that accurate metrology of $10 \AA$ gate oxides (i.e. limited to several per cent error) is feasible on a consistent basis using a $\mathrm{C}_{\mathrm{s}}$-corrected microscope. However, the effects of $\mathrm{Si}_{-} \mathrm{SiO}_{2}$ interface roughness on 
measurement accuracy should be explored in greater detail to assess the applicability of zero- $\mathrm{C}_{\mathrm{s}}$ microscopy to metrology of real systems.

\section{Acknowledgments}

This work was supported by the Intel Corporation, and by the Director, Office of Energy

Research, Office of Basic Energy Sciences, Material Sciences Division of the U.S. Department of Energy, under contract No. DE-AC03-76SF00098. Access to the computing facility at the National Center for Electron Microscopy is gratefully acknowledged. The authors wish to thank Dr. Roar Kilaas at NCEM for helpful instruction with the image simulation software. 


\section{$\underline{\text { References }}$}

Cowley JM, Moodie AF (1957) The scattering of electrons by atoms and crystals. I. A new theoretical approach. Acta Cryst 10:609-619

Goodman P, Moodie AF (1974) Numerical evaluation of n-beam wave functions in electron scattering by the multi-slice method. Acta Cryst Sect A 30:280-290

Haider M, Rose H, Uhlemann S, Schwan E, Kabius B, Urban K (1998) A spherical-aberrationcorrected $200 \mathrm{kV}$ transmission electron microscope. Ultramicroscopy 75:53-60 Jamison R (2000) Computational and Experimental Quantification of Focused Ion Beam Damage in Silicon During TEM Specimen Preparation. Ph.D. Dissertation, University of California, Berkeley, CA Lynch DF (1971) Out-of-zone effects in dynamical electron diffraction intensities from Au. Acta Cryst Sect A 27:399-407

O’Keefe MA, Kilaas R (1988) Advances in high resolution image simulation. Scanning Microsc Suppl 2:225-244

O'Keefe MA, Dahmen U, Hetherington CJD (1989) Simulated image maps for use in experimental high-resolution electron microscopy. In: Atomic Scale Structure of Interfaces, Bringans RD, Feenstra RM, Gibson JM (eds). Materials Research Society Symposium Proceedings Vol. 159. Pittsburgh: MRS, pp 453-458

O’Keefe MA, Radmilovic V (1992) Extension of the thin crystal condition by small crystal tilts: why HREM images of SiC polytypes always look tilted. In: Proceedings $-50^{\text {th }}$ Annual Meeting of Electron Microscopy Society of America, Bailey GW, Bentley J, Small JA (eds). San Francisco: San Francisco Press, pp 116-117 
O’Keefe MA, Radmilovic V (1993) The effects of small crystal tilts on dynamical scattering: why simulated images are thinner than experimental ones. In: Proceedings $-51^{\text {st }}$ Annual Meeting of Microscopy Society of America, Bailey GW, Reider CL (eds). San Francisco: San Francisco Press, pp 980-981

O’Keefe MA (1998) High resolution transmission electron microscopy at zero $\mathrm{C}_{\mathrm{s}}$. In: Proceedings - Microscopy and Microanalysis 1998, Bailey GW, Alexander KB, Jerome WG, Bond MG, McCarthy JJ (eds). Atlanta: Springer, pp 382-383

Urban K, Kabius B, Haider M, Rose H (1999) A way to higher resolution: spherical-aberration correction in a $200 \mathrm{kV}$ transmission electron microscope. J Electron Microsc 48:821-826 
Table 1. Optimum defocus conditions and resulting point-to-point resolutions for different $C_{s}$ values, calculated for JEOL 2010F HRTEM.

\begin{tabular}{|c|c|c|}
\hline $\mathrm{C}_{\mathrm{s}}(\mathrm{mm})$ & Scherzer Defocus $(\AA)$ & Resolution $(\AA)$ \\
\hline 1.0 & -601 & 2.29 \\
\hline 0.5 & -425 & 1.92 \\
\hline 0.2 & -269 & 1.54 \\
\hline 0.05 & -134 & 1.09 \\
\hline 0.0 & -20 & 1.00 \\
\hline
\end{tabular}


Table 2. Summary of measurement errors for various microscope and specimen variables.

\begin{tabular}{|c|c|c|c|c|c|c|c|}
\hline $\begin{array}{c}\text { Experiment } \\
\text { No. }\end{array}$ & $\begin{array}{c}\text { Specimen } \\
\text { Thickness } \\
(\AA)\end{array}$ & $\begin{array}{c}\text { Specimen } \\
\text { Tilt } \\
(\mathrm{mrad})\end{array}$ & $\begin{array}{c}\text { Defocus } \\
(\AA)\end{array}$ & $\begin{array}{c}\mathrm{C}_{\mathrm{s}} \\
(\mathrm{mm})\end{array}$ & $\begin{array}{c}\text { Model } \\
\text { Thickness } \\
(\AA)\end{array}$ & $\begin{array}{c}\text { Measured } \\
\text { Thickness } \\
(\AA)\end{array}$ & $\%$ Error \\
\hline 1 & 154 & 0 & -425 & 0.5 & 10.56 & 9.84 & -6.8 \\
\hline 2 & 154 & 0 & -156 & 0.5 & 10.56 & 11.40 & +8.0 \\
\hline 3 & 154 & 0 & -20 & 0.0 & 10.56 & 10.44 & -1.1 \\
\hline 4 & 154 & 0 & -269 & 0.2 & 10.56 & 9.60 & -9.1 \\
\hline 5 & 154 & 0 & -601 & 1.0 & 10.56 & 10.80 & +2.3 \\
\hline 6 & 154 & 6.3 & -425 & 0.5 & 10.56 & 9.72 & -8.0 \\
\hline 7 & 154 & 12.6 & -425 & 0.5 & 10.56 & 9.12 & -13.6 \\
\hline 8 & 154 & 25 & -425 & 0.5 & 10.56 & 10.68 & +1.1 \\
\hline 9 & 154 & 6.3 & -20 & 0.0 & 10.56 & 10.56 & 0.0 \\
\hline 10 & 154 & 12.6 & -20 & 0.0 & 10.56 & 10.68 & +1.1 \\
\hline 11 & 246 & 0 & -425 & 0.5 & 10.56 & 8.88 & -15.9 \\
\hline 12 & 246 & 0 & -156 & 0.5 & 10.56 & 10.92 & +3.4 \\
\hline 13 & 246 & 0 & -20 & 0.0 & 10.56 & 10.56 & 0.0 \\
\hline 14 & 246 & 3.0 & -425 & 0.5 & 10.56 & 9.24 & -12.5 \\
\hline 15 & 246 & 6.3 & -425 & 0.5 & 10.56 & 9.48 & -10.2 \\
\hline 16 & 246 & 12.6 & -425 & 0.5 & 10.56 & 9.60 & -9.1 \\
\hline 17 & 246 & 25 & -425 & 0.5 & 10.56 & 9.00 & -14.8 \\
\hline
\end{tabular}


Figure Captions

Figure 1. (a) Model device structure viewed in cross-section depicts orientation of substrate, gate electrode, and gate oxide containing two different classes of defects. Simulated images are formed by slicing the device structure in the beam direction to form a series of phase gratings, as illustrated in (b).

Figure 2. Actual gate oxide thickness, T, derived from the model structure (a) is compared to the thickness, $t$, measured from the simulated image (b). A histogram of standard deviation in pixel intensity (c) can be used to precisely locate the boundaries of the gate oxide in the simulated image.

Figure 3. Simulated images of cylindrical Si[110] defects embedded in a $16 \AA$ gate oxide. Defect visibility decreases as the cylinder diameter decreases from (a) $20 \AA$ to (b) $10 \AA$. Arrows depict the anticipated defect location consistent with model structure. Total specimen thickness is $138 \AA$.

Figure 4. Simulated images of hemispherical Si[110] defects in a16 A gate oxide. Defect visibility decreases as the hemisphere radius varies from (a) $16 \AA$ to (b) $13 \AA$ to (c) $10 \AA$, at which point the defect is no longer visible. Arrows depict the anticipated defect location consistent with model structure. Total specimen thickness is $123 \AA$.

Figure 5. Simulated images of hemispherical surface defects having orientations (a) $\mathrm{Si}[111]$ and (b) $\mathrm{Si}[100]$. Arrows depict the anticipated defect location consistent with model structure. Defect radius is $16 \AA$ and the total specimen thickness is $123 \AA$.

Figure 6. Simulated images of $\mathrm{Si}[111]$ hemispherical surface defects using reduced $\mathrm{C}_{\mathrm{s}}$ values of (a) $0.2 \mathrm{~mm}$, (b) $0.05 \mathrm{~mm}$ and (c) $0.0 \mathrm{~mm}$. Defect visibility is greatly enhanced at $\mathrm{C}_{\mathrm{s}}=0.0 \mathrm{~mm}$, 
where the hexagonal arrangement of $\mathrm{Si}[111]$ atoms can be clearly observed (d). Defect radius is $16 \AA$ and the total specimen thickness is $123 \AA$.

Figure 7. Simulated images of $\mathrm{Si}[100]$ hemispherical surface defects using reduced $\mathrm{C}_{\mathrm{s}}$ values of (a) $0.2 \mathrm{~mm}$, (b) $0.05 \mathrm{~mm}$ and (c) $0.0 \mathrm{~mm}$. Negligible improvements in defect visibility are observed with decreasing $\mathrm{C}_{\mathrm{s}}$. Defect radius is $16 \AA$ and the total specimen thickness is $123 \AA$. Figure 8. Plot of measurement error versus specimen tilt angle for $154 \AA$ (circles) and $246 \AA$ (squares) specimen thickness reveal no consistent trends in measurement accuracy as a function of tilt.

Figure 9. Plot of measurement error versus spherical aberration coefficient shows no obvious trend for $154 \AA$ specimen thickness.

Figure 10. Simulated images of $10 \AA$ gate oxide structure using $C_{s}=0.0 \mathrm{~mm}$. At (a) $154 \AA$ and (b) $246 \AA$ specimen thickness, the model structure is faithfully reproduced and the gate oxide thickness can be measured accurately to within $1 \%$ error.

Figure 11. Plot of measurement error versus specimen tilt angle for $\mathrm{C}_{\mathrm{s}}$-corrected images demonstrates that gate oxide thickness can be measured accurately to within $1 \%$ error even at significant specimen tilt. Total specimen thickness is $154 \AA$. 


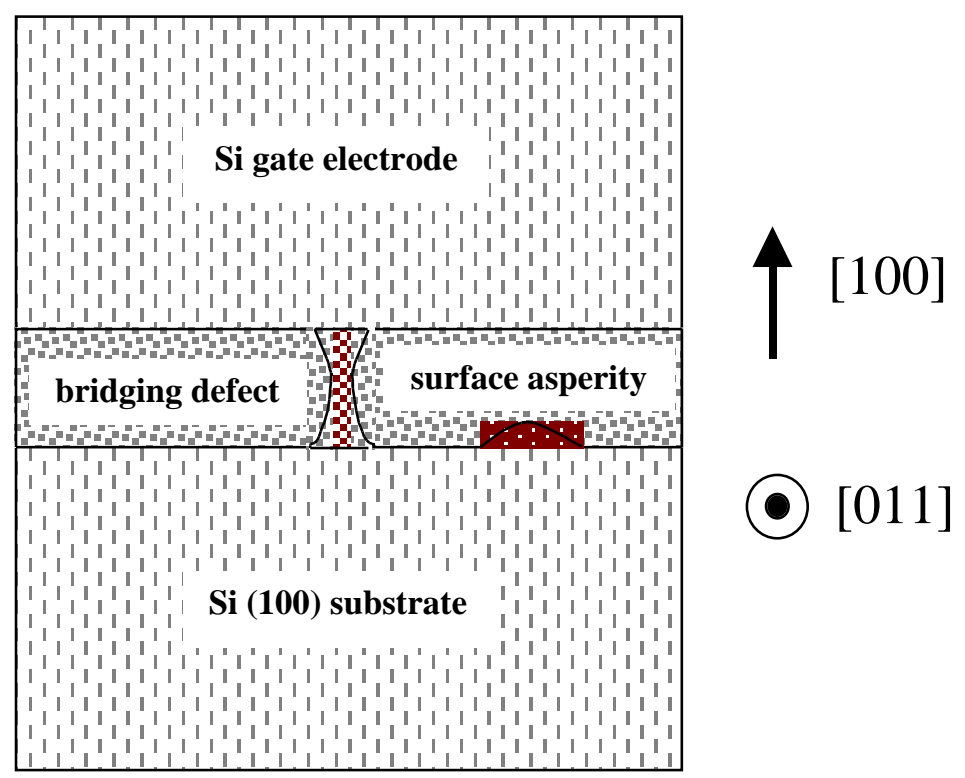

(a)

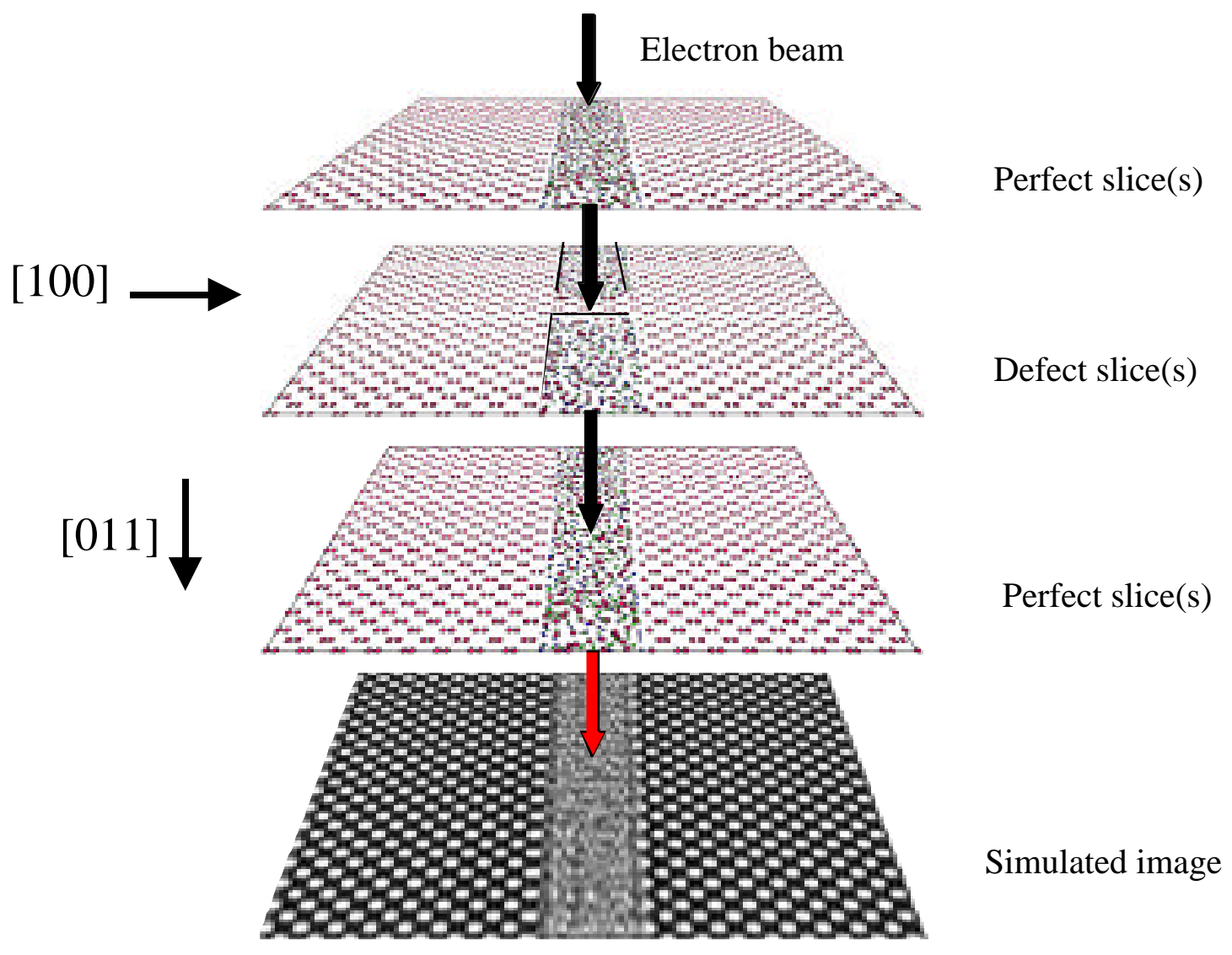

(b) 


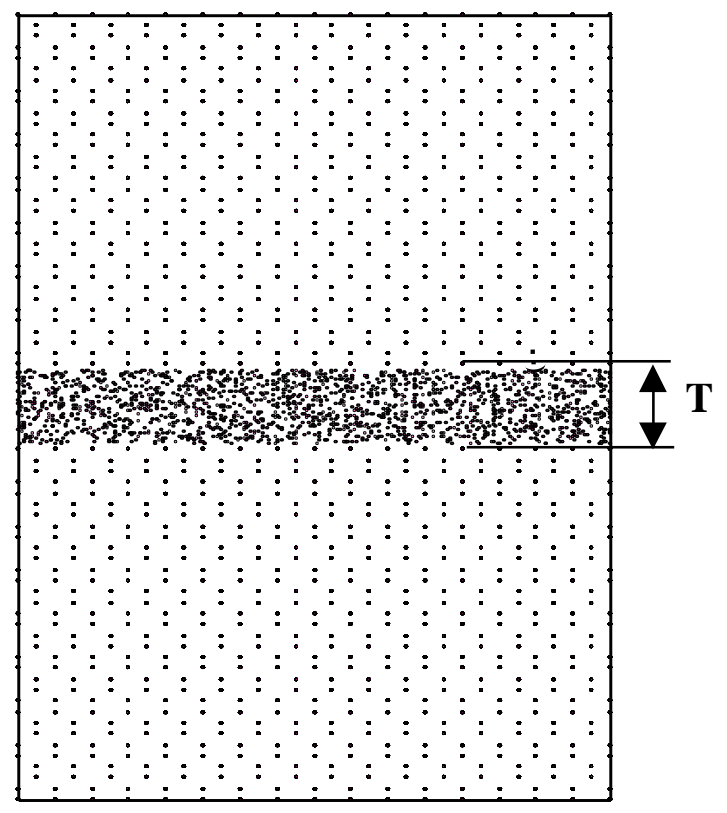

(a)

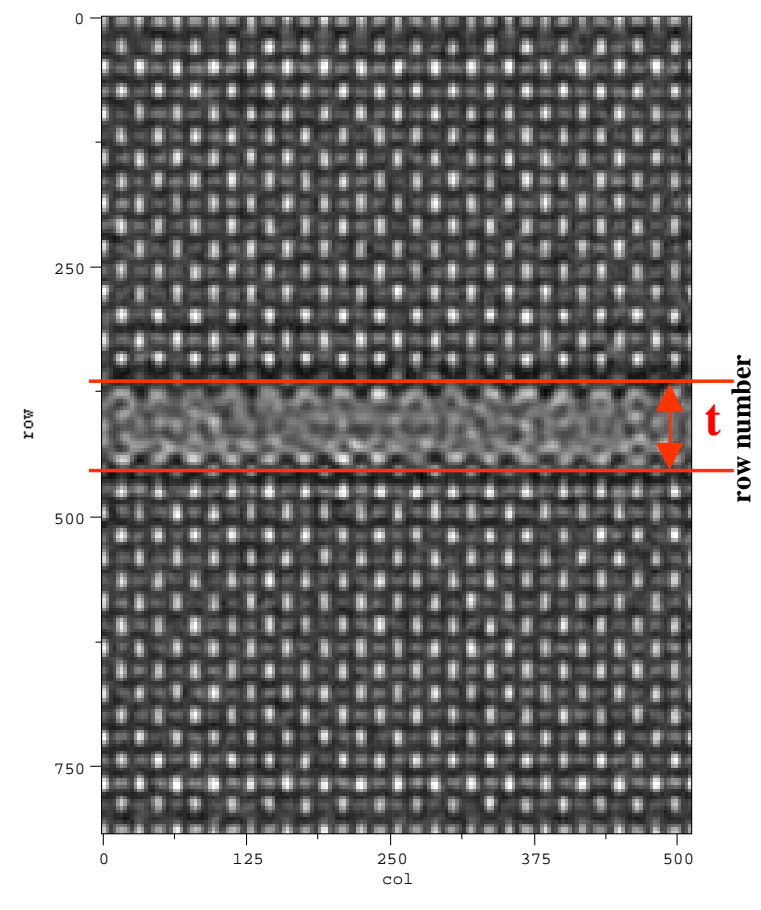

(b)

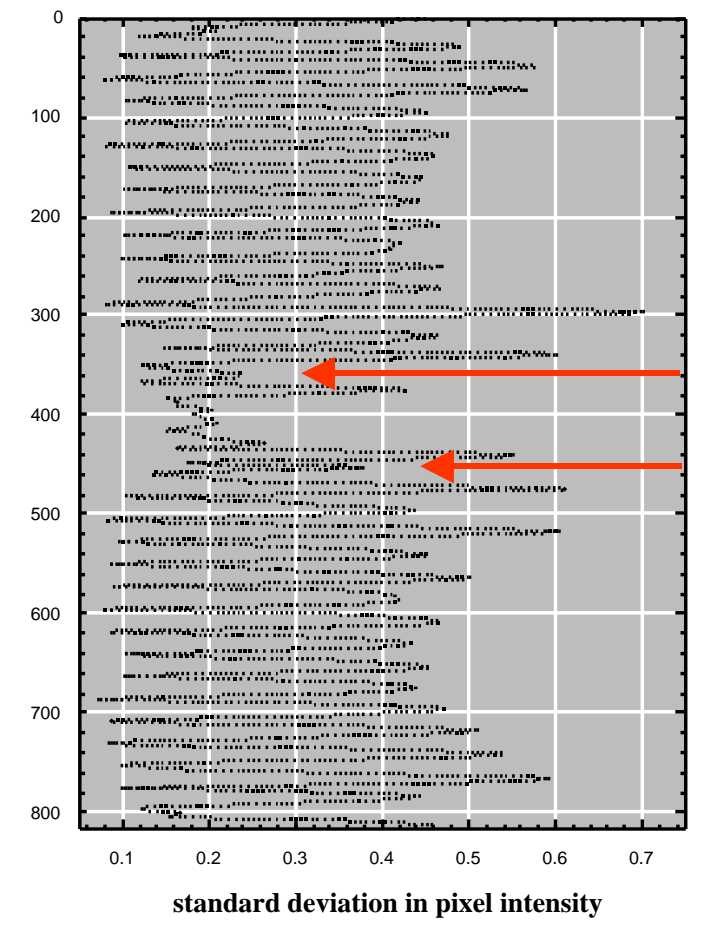

(c) 


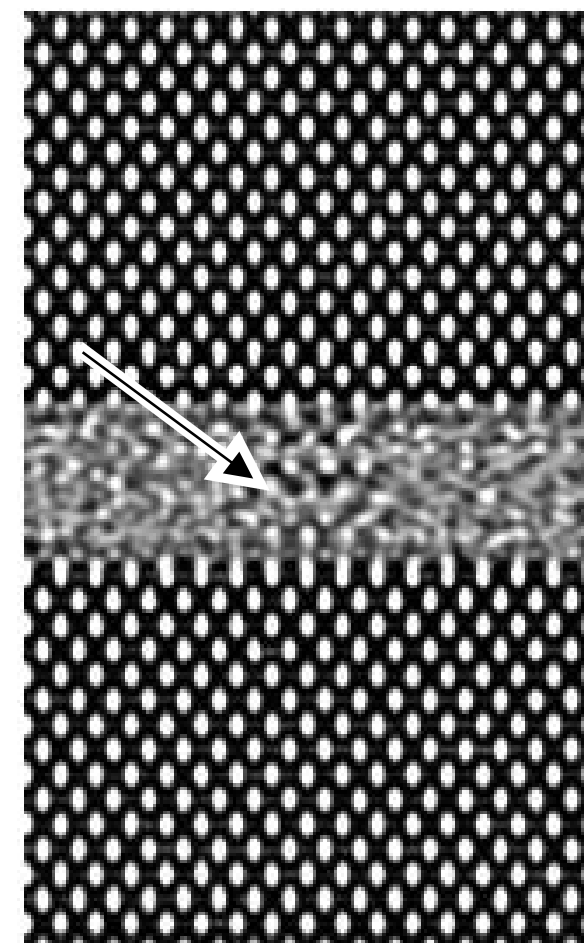

(a)

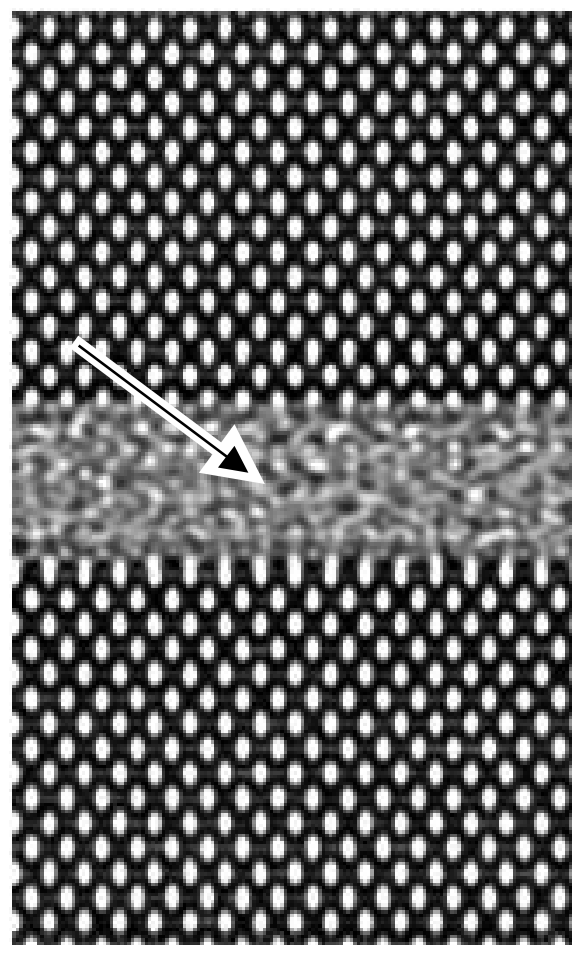

(b) 


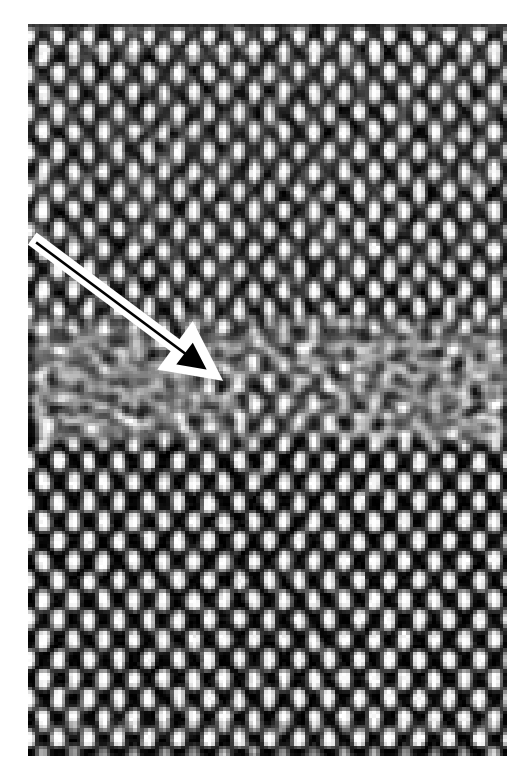

(a)

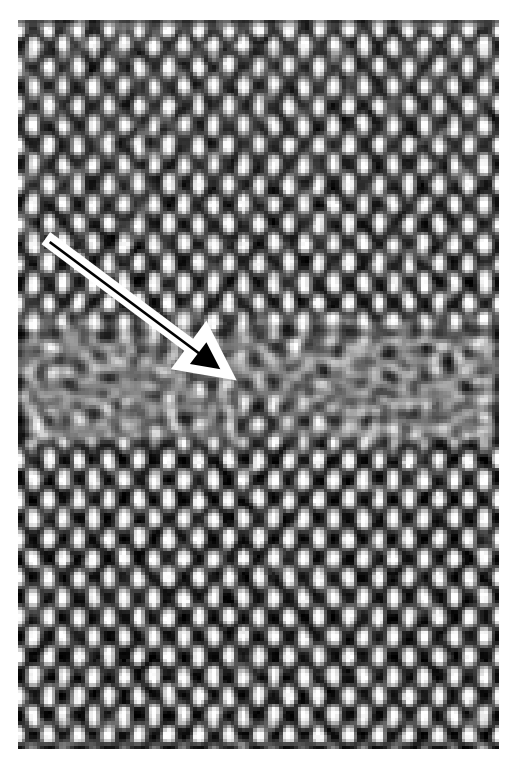

(b)

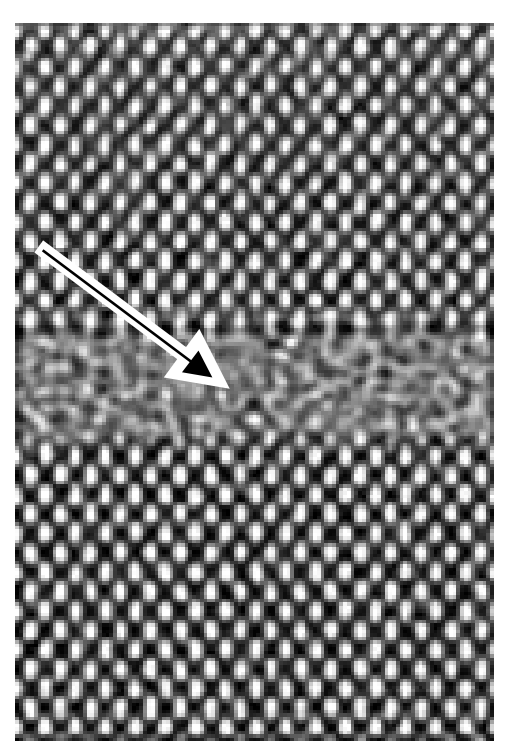

(c) 


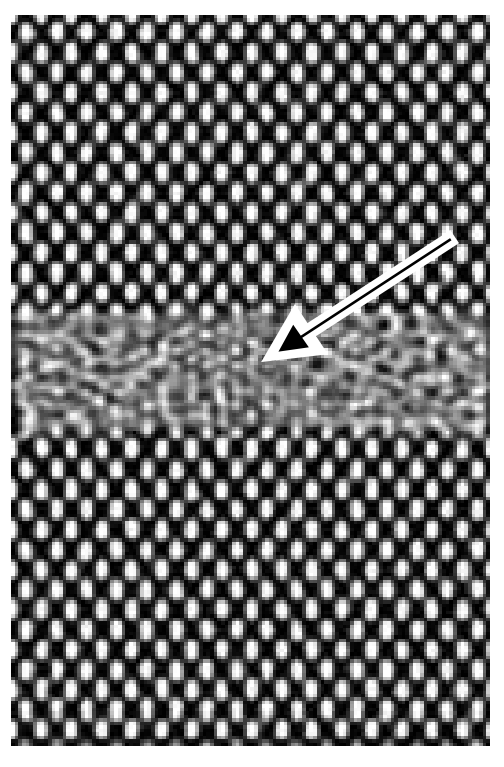

(a)

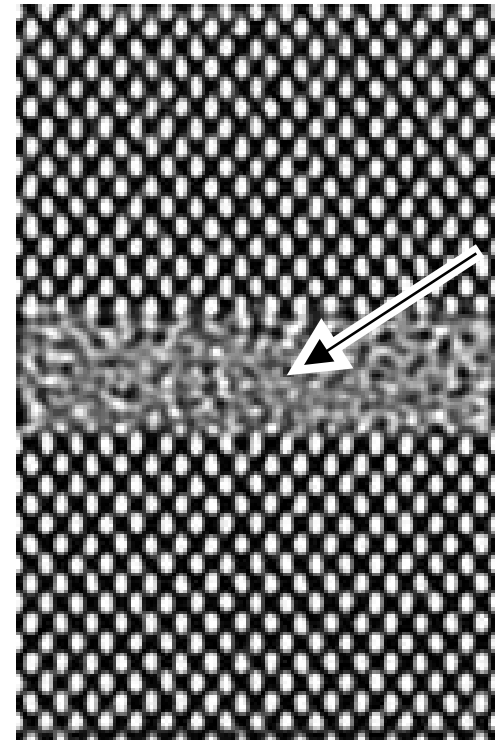

(b) 


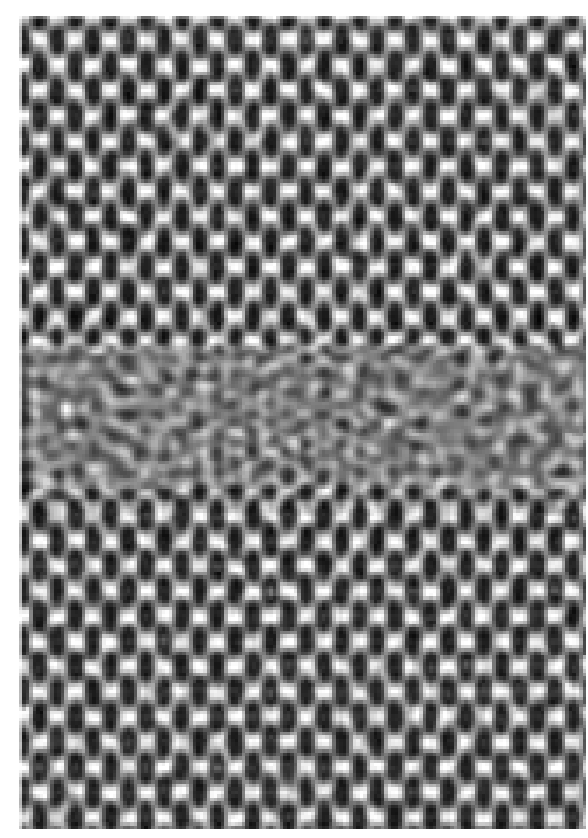

(a)

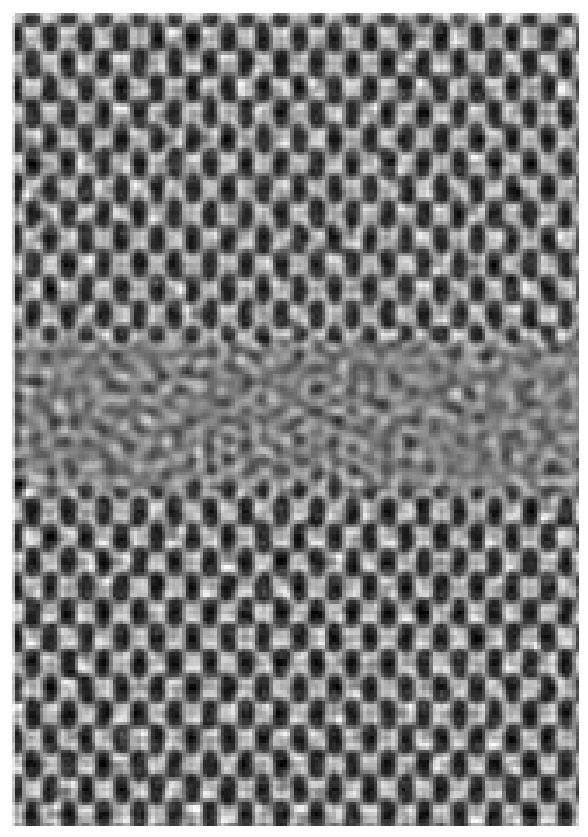

(b)

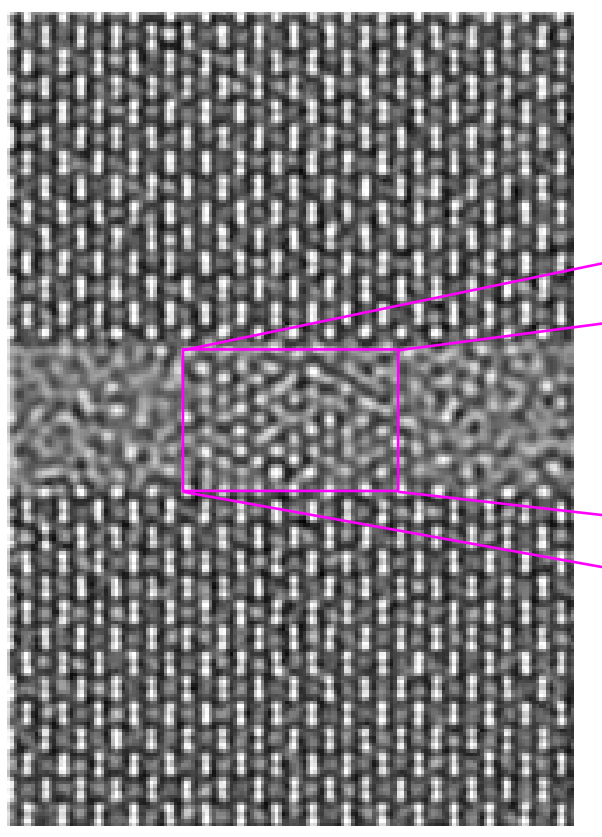

(c) 


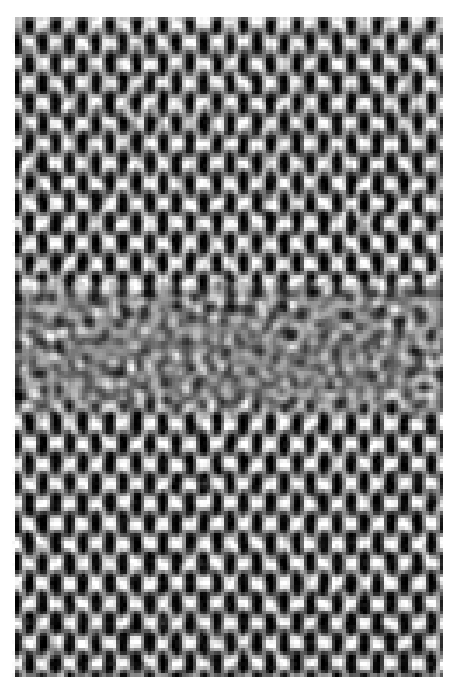

(a)

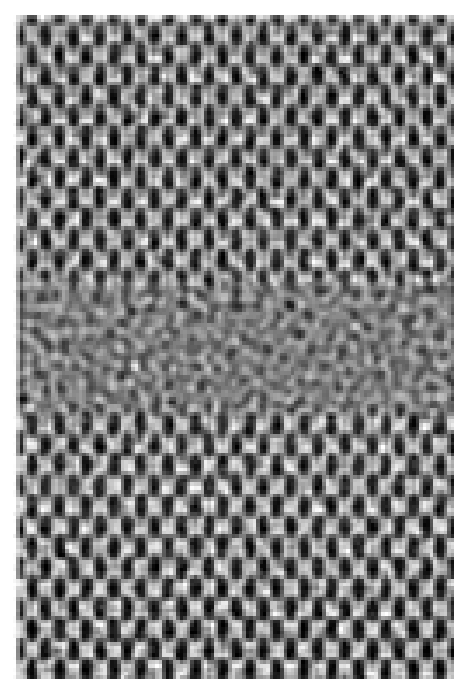

(b)

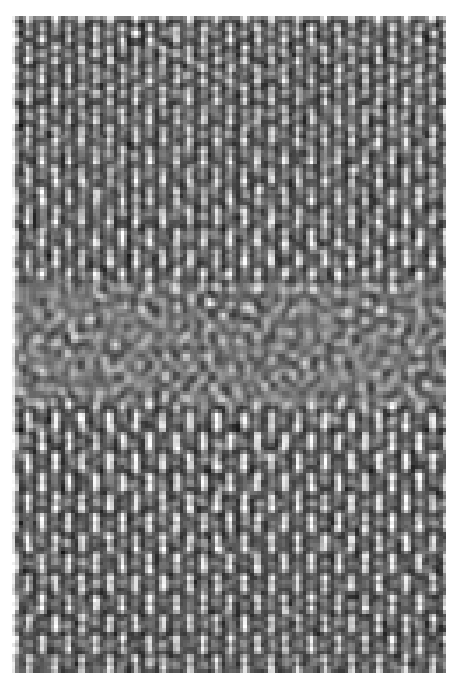

(c) 


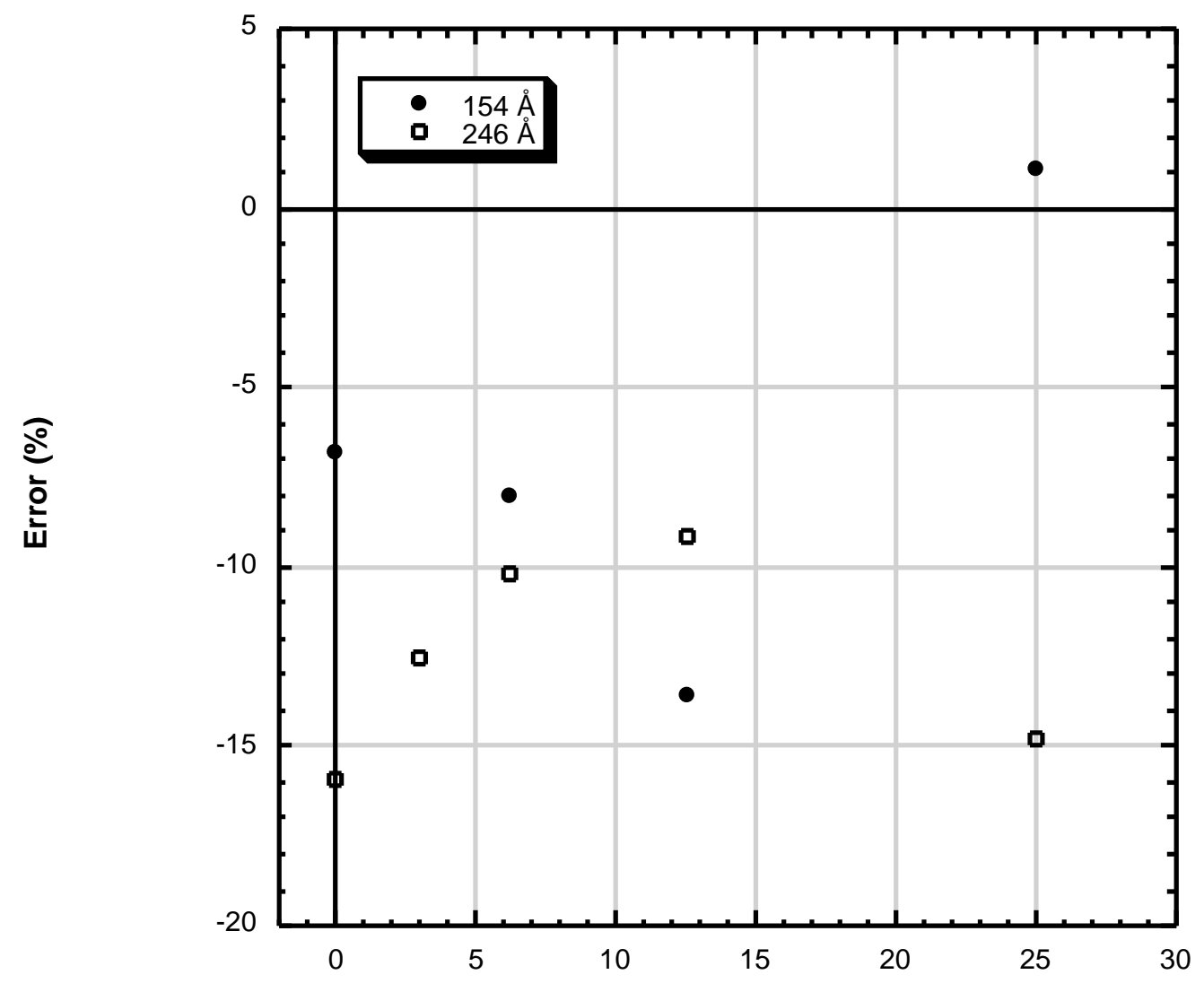

Tilt Angle (mrad) 


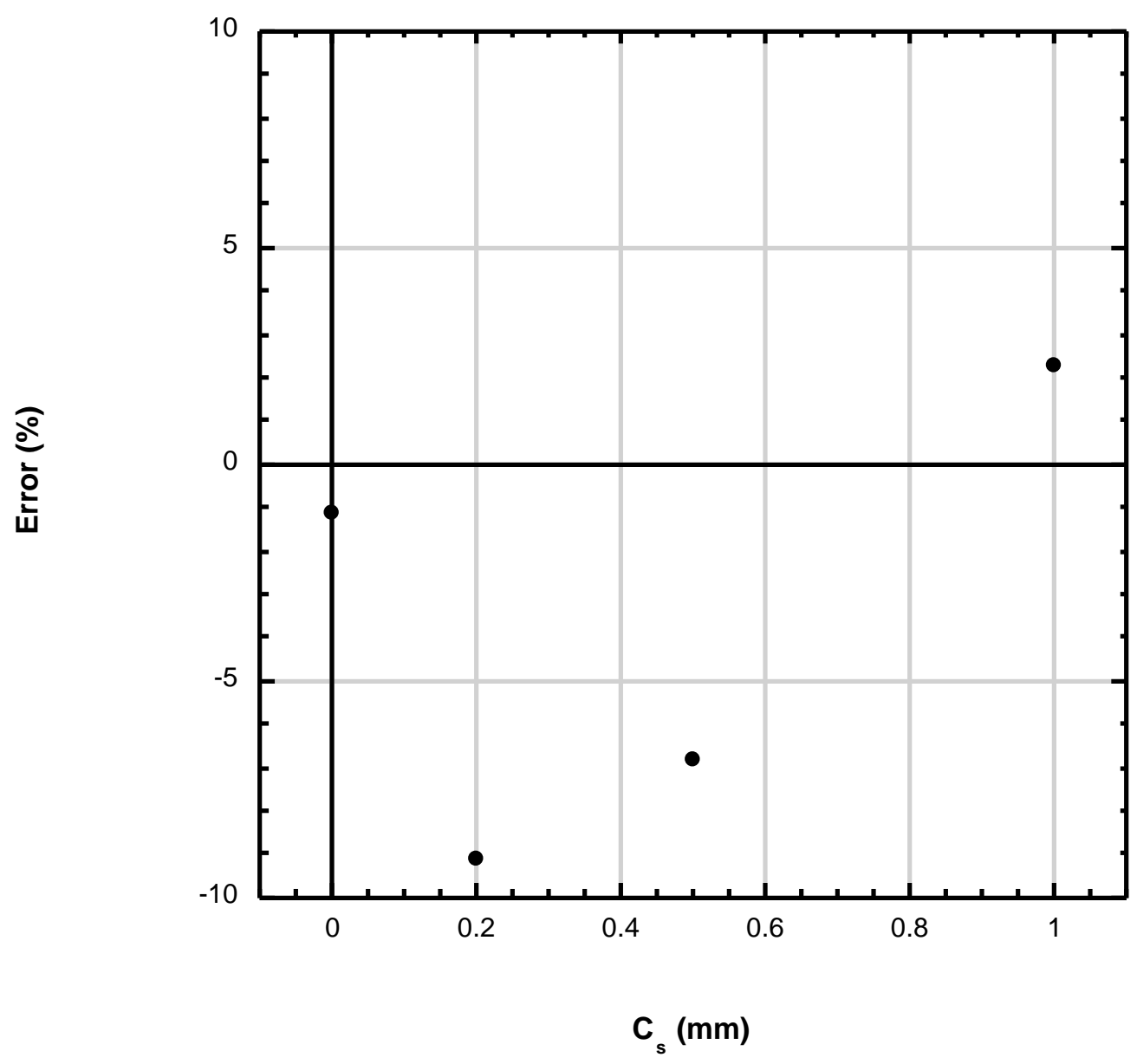




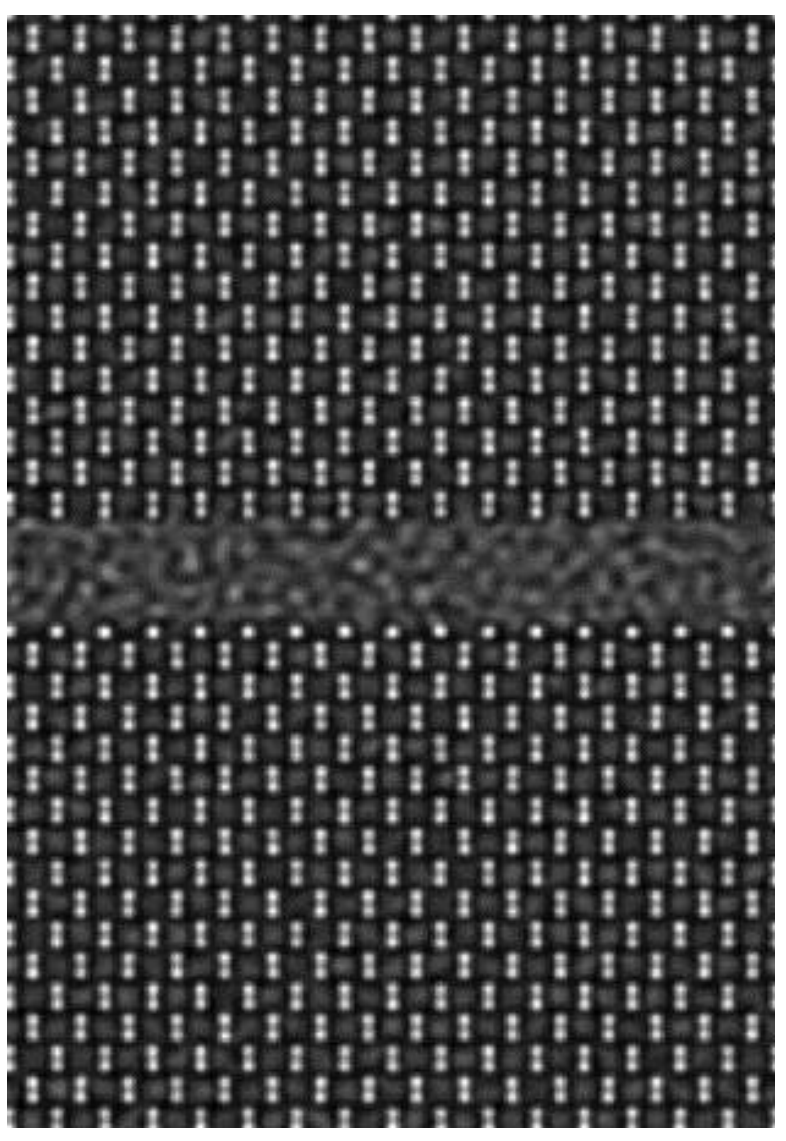

(a)

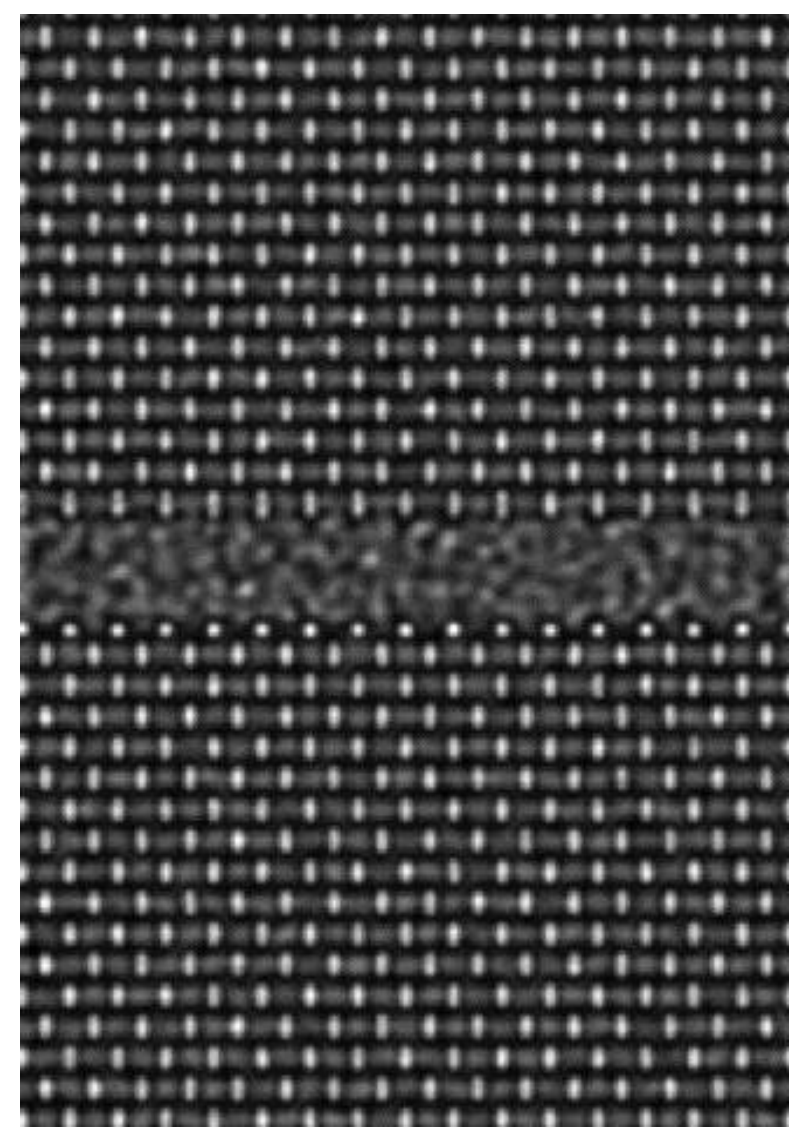

(b) 


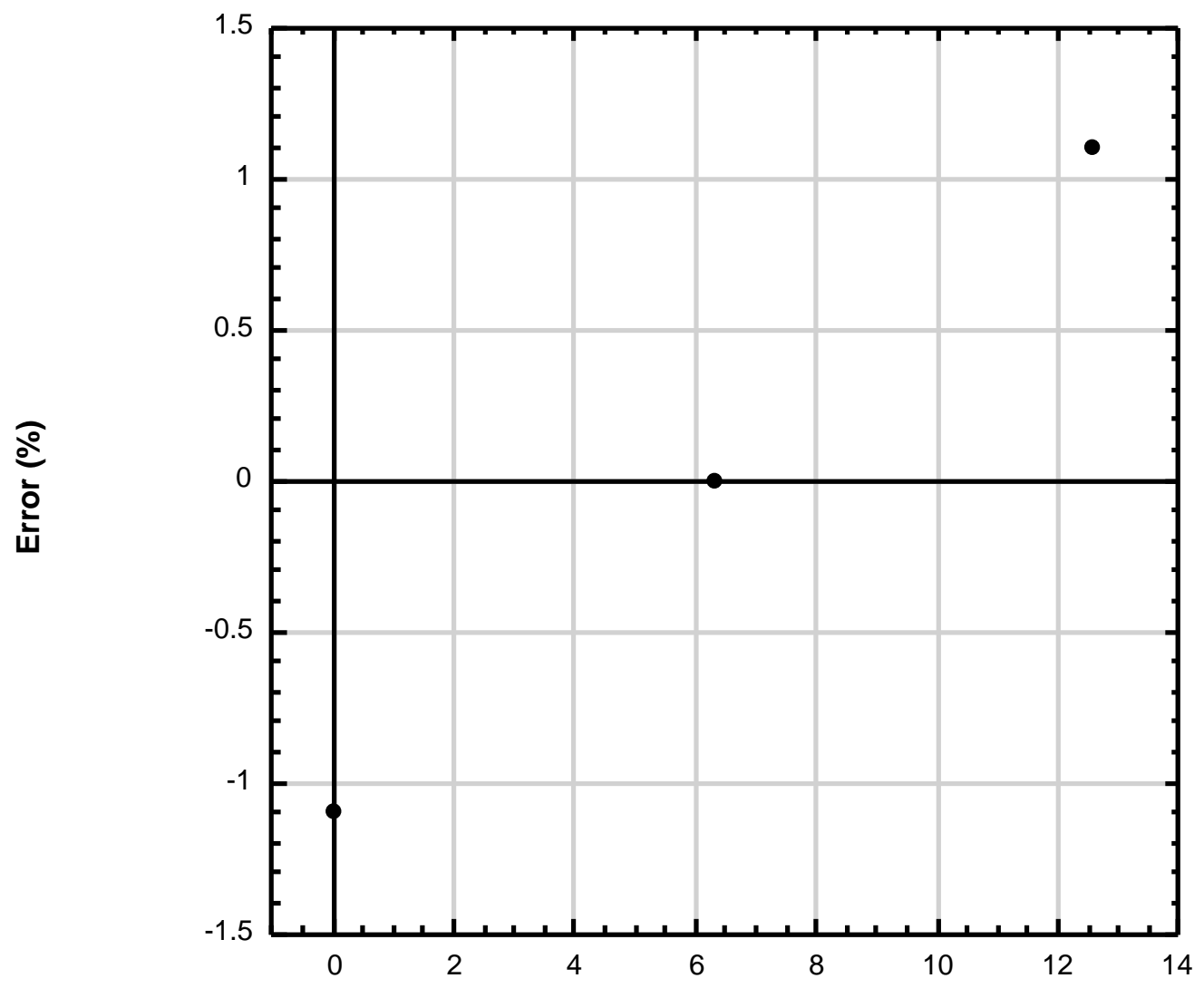

Tilt Angle (mrad) 